Check for updates

Cite this: RSC Adv., 2017, 7, 53322

Received 4th August 2017

Accepted 25th October 2017

rsc.li/rsc-advances
DOI: $10.1039 / c 7 r a 08625 c$

\section{The dual-hit metabolic modulator LDCA synergistically potentiates doxorubicin to selectively combat cancer-associated hallmarks $\dagger$}

\author{
Suchandrima Saha, \$ Monisankar Ghosh $\ddagger$ and Samir Kumar Dutta (D) *
}

Conventional chemotherapy induces significant toxicity, leading to serious side effects; thus, an effective strategy to counter this would be to use a combination of drugs that have unrelated mechanisms of action and drug resistance. Doxorubicin, a widely used chemotherapeutic drug, has adverse side effects; thus, it is crucial to reduce its toxicity to improve its therapeutic regimen. Cancer cells vitally differ from normal cells with respect to their metabolism, and the use of metabolic modulators is expected to proximally compensate the various manifestations of cancer. In this study, doxorubicin was used in combination with a dual-hit metabolic modulator, LDCA, with the postulation that this adroit targeting of cancer cells would exert potent therapeutic effects. The results demonstrated that this combination synergistically enhanced the growth inhibition and induced mitochondria-mediated apoptosis by recruiting the caspase cascade, restricting migration, and obviating the clonogenic outgrowth potential of melanoma cells. Interestingly, the combination specifically dampened melanoma cell viability, but spared the normal population; this suggested its low toxicity profile. Furthermore, in the preclinical model of murine melanoma, combination treatment thwarted tumor growth kinetics to restrain oncogenic progression, thus accentuating survival. Comprehensively, we have demonstrated the promising therapeutic potential of the combination of doxorubicin and LDCA in the expanding chapter of combinatorial therapy.

\section{Introduction}

Most of the common human cancers are malignant neoplasms of the skin, and despite significant efforts, they remain poorly understood at the molecular level and fail to respond to conventional chemotherapy. ${ }^{\mathbf{1 - 4}}$ Therefore, patients harboring this recurrent disease highlight the unmet need and importance of new anticancer agents that would replace and/or supplement the therapeutic options available currently. ${ }^{4}$

Doxorubicin (Adriamycin) is one of the most effective clinical chemotherapeutic drugs developed that garners a broad spectrum of therapeutic antineoplastic activity against various cancers. $^{5-7}$ However, its efficacy against melanoma is limited partly because of its inability to attain optimum physiological concentration without systemic toxicity and also plausibly due to the phenomenon of drug resistance. ${ }^{8-11}$ Although the

Drug Development Diagnostics and Biotechnology, CSIR-Indian Institute of Chemical Biology (CSIR-IICB), 4 Raja S. C. Mullick Road, Kolkata-700032, West Bengal, India. E-mail: suchandrima.saha5@gmail.com; monisankarghosh88@gmail.com; somir_india@yahoo.com; Fax: +91-33-24735197; +91-33-24723967; Tel: +91-3324995701

$\dagger$ Electronic supplementary information (ESI) available. See DOI: 10.1039/c7ra08625c

† S. S. and M. G. contributed equally to this work. mechanisms responsible for the development of primary drug resistance still remain to be elucidated, a verifiable therapy that would lead to disease remission is of significant need.

Malignant cells exhibit metabolic alterations as compared to the normal cells; moreover, although this metabolic reprogramming has been recently designated as yet another general hallmark of cancer, accumulating evidence suggests that metabolic alterations are intimately associated with and allow the varied facets of malignancy. ${ }^{12-14}$ During the past decade, decoding of the intimate relationship between metabolism and malignancy has been the main challenge in tumor biology; ${ }^{15-17}$ therefore, targeting cancer metabolism is considered a key strategy for the development of selective antineoplastic agents. ${ }^{18-23}$

Moreover, one of the enzymes intricately linked with altered metabolism is LDH-A, which plays an important role in fueling aerobic glycolysis in cancer cells; ${ }^{\text {24,25 }}$ thus, targeting LDH-A can consequentially obviate several cancer-acquired hallmark features. ${ }^{26,27}$ A dual-hit metabolic modulator, LDCA, recently developed by us, showed potent anti-proliferative activity, specifically against cancer cells where it competitively inhibited LDH-A activity, subsequently altering mitochondrial hyperpolarization and concomitantly subverting apoptosis specifically in the malignant population, without manifesting toxicity. ${ }^{28}$ Indeed, with a clear safety profile, we extended our studies to 
delineate its effect in a preclinical model, and the results suggested the potential use and prospectus of LDCA in a clinical setting. ${ }^{29}$

Although new drugs are continually being discovered, there are problems that arise partly because agents gated for targeting at specific points frequently show limited efficacy that leads to cellular adaptivity, poor safety, and resistance profiles. ${ }^{30-32}$ Considering these issues, treatment with multicomponent therapies and multicomponent agents is being increasingly emphasized as a potentially more productive strategy; thus, efforts are being directed towards the development of these rationally designed therapeutic regimens. When administered in combination, therapies are expected to become essentially more effective and produce pharmacokinetically potent activity that would lead to reduced dosage and minimized overlapping cytotoxicity without unacceptable si†de-effects and drug resistance. ${ }^{33-35}$

Evidence imply that therapeutic benefits are maximized when agents with different mechanisms of action are combined; therefore, we have postulated that since LDCA specifically blocks the LDH-A enzyme activity and effectively alters malignant mitochondrial bioenergetics, its combination with doxorubicin at a calculated dosage would potentially limit melanoma progression. The results suggestively delineated that the selected combination of doxorubicin and LDCA significantly garnered the synergistic antitumor modality by subverting apoptosis selectively in the melanoma population and importantly by maintaining a low toxicity profile. We further ventured to understand the combinatorial synergistic potential of doxorubicin and LDCA in the preclinical model of murine melanoma, and in vivo studies comprehensively elaborated the effectiveness of this combination; this highlights the application potential of this combination in the clinical setting.

\section{Experimental}

\section{Chemicals and reagents}

Doxorubicin was purchased from Sigma Aldrich, and LDCA was synthesized, purified, and characterized as previously described (Fig. 1A). ${ }^{28}$ Dimethyl sulphoxide (DMSO), paraformaldehyde, Dulbecco's Modified Eagle's Medium (DMEM), bovine serum albumin (BSA), and Histopaque-1077 were purchased from Sigma (St. Louis, Missouri, USA). Pen Strep, MTT (3-[4,5dimethylthiazol-2-yl]-2,5- diphenyltetrazolium bromide), $5,5^{\prime}, 6,6^{\prime}$-tetrachloro-1,1',3,3'- tetraethyl benzimidazolylcarbocyanine iodide (JC-1), 4',6-diamidino-2-phenylindole (DAPI), CellRox Deep Red reagent, MitoTracker Deep Red, rhodaminephalloidin, and fetal bovine serum (FBS) were obtained from Thermo Fisher Scientific (Waltham, MA, USA). The antibodies anti-Cyt c, anti-cleaved caspase-3, anti-cleaved caspase-9, anticleaved PARP, and PCNA anti- $\beta$-actin were purchased from Cell Signalling Technologies (Beverly, MA). Other reagents were of analytical grade.

\section{Cell culture}

B16-F10 (mouse skin cancer) and WI-38 (lung fibroblast) cells were obtained from the National Centre For Cell Science
(NCCS), Pune, Maharashtra, India, and cultured in a complete DMEM medium at $37{ }^{\circ} \mathrm{C}$ with $5 \% \mathrm{CO}_{2}$. Cells from exponentially growing cultures were used for all the experiments.

Doxorubicin hydrochloride was dissolved in PBS, and LDCA was dissolved in DMSO and diluted in PBS such that the final concentration of DMSO was not more than $2 \%$, which was well tolerated by the mice and used accordingly for experiments.

\section{Cell viability analysis}

The cytotoxic potential of doxorubicin and LDCA was assessed using the MTT assay. Briefly, B16-F10 and WI-38 cells $\left(1 \times 10^{4}\right)$ were seeded in $96=$ well plates and treated with doxorubicin (10-500 $\mathrm{nM})$ and LDCA $(2-100 \mu \mathrm{M})$ alone or in combination for $72 \mathrm{~h}$. Then, $20 \mu \mathrm{l}$ of $5 \mathrm{mg} \mathrm{ml}^{-1}$ MTT was added, and absorbance was measured at $570 \mathrm{~nm}$ using a Thermo MULTISKAN FC plate reader.

\section{Flow cytometry}

Cells were treated with $500 \mathrm{nM}$ doxorubicin, $20 \mu \mathrm{M}$ LDCA, and their combination for $24 \mathrm{~h}$ and then harvested, resuspended in $400 \mu \mathrm{l}$ of binding buffer, and incubated with Annexin-V FITC and PI for estimating apoptosis. Data were acquired using a BD LSR Fortressa II flow cytometer and analyzed using the FACS DIVA 6.2 software.

\section{Soft agar colony formation assay}

In the soft agar colony formation assay, $10^{4}$ cells were mixed in a $0.35 \%$ agarose complete medium and plated on a $0.7 \%$ agar complete medium in $35 \mathrm{~mm}$ plates. The media containing vehicle, $500 \mathrm{nM}$ doxorubicin, $20 \mu \mathrm{M}$ LDCA, and their combination were changed every alternate day for 2 weeks. Phase contrast images were obtained from 10 different fields using a Nikon Ti microscope $(20 \times)$.

\section{Micronuclei (MN) formation and chromosomal aberration (CA) study}

The experiments were approved by the Human Ethics Committee of the CSIR-Indian Institute of Chemical Biology and performed in concordance with the institutional ethics committee guidelines. Informed consent was obtained from human subjects to perform the experiments. Lymphocytes from human blood samples were separated using ficoll-gradient centrifugation and cultured in RPMI-1640, followed by incubation with a combination of $10 \mu \mathrm{M}$ doxorubicin and $20 \mu \mathrm{M}$ LDCA for $72 \mathrm{~h}$. For the CA study, $0.04 \%$ of colchicine was added to the culture during the last $2 \mathrm{~h}$ of incubation as a positive control, and in the case of micronuclei formation, $6 \mu \mathrm{g} \mathrm{ml}{ }^{-1}$ of cytochalasin B was added $4 \mathrm{~h}$ before harvesting the cells. Slides were prepared and stained with Giemsa. For the chromosomal aberration study, the mitotic index was deduced at $100 \times$ and expressed in percentage. For $\mathrm{MN}$ detection, 1000 cells from each slide were scored at $60 \times$.

\section{Measurement of mitochondrial membrane potential using JC-1}

Cells were cultured on coverslips overnight, treated with $500 \mathrm{nM}$ doxorubicin, $20 \mu \mathrm{M}$ LDCA, either alone or in combination for 
$16 \mathrm{~h}$ at $37^{\circ} \mathrm{C}$, washed with PBS, and incubated with $10 \mu \mathrm{g} \mathrm{ml}$ of JC- 1 at $37^{\circ} \mathrm{C}$ for $15 \mathrm{~min}$. Finally, the cells were analysed using a confocal laser scanning microscope (CLSM) at $40 \times$. For flow cytometric analysis, pre-treated cells were harvested, processed as abovementioned, and data were acquired using the BD LSR Fortressa II flow cytometer and analyzed using the FACS DIVA 6.2 software.

\section{Immunocytochemistry}

Cells were seeded on coverslips, incubated at $37{ }^{\circ} \mathrm{C}$ with $5 \%$ $\mathrm{CO}_{2}$, and treated with a combination of $500 \mathrm{nM}$ doxorubicin and $20 \mu \mathrm{M}$ LDCA for $16 \mathrm{~h}$. Cells were fixed using $4 \%$ paraformaldehyde and permeabilized with $0.2 \%$ Triton $\mathrm{X}-100$ for 15 min on ice. After blocking in 1\% BSA for one hour at room temperature, cells were incubated overnight with primary antibodies against Cyt c, cleaved caspase-9/3 and PARP, and acetylated tubulin $(1: 200)$ at $4{ }^{\circ} \mathrm{C}$. Subsequently, coverslips were incubated with Alexa Fluor-488 or Alexa Fluor-555conjugated secondary antibody $(1: 1000)$ in $1 \%$ BSA at room temperature for $2 \mathrm{~h}$. For the detection of actin fibers, cells were incubated with rhodamine-labeled phalloidin for $20 \mathrm{~min}$. The nucleus was stained using DAPI. Finally, coverslips were washed in PBS and examined under CLSM at $40 \times$. Images were obtained and processed using the Andor iQ2 software and analyzed using the Image software.

\section{Immunohistochemistry}

Tissue samples were fixed in $10 \%$ formalin and immersed successively in sucrose gradients of $15 \%$ and $30 \%$ until tissue sunk to the bottom. Then, $8 \mu \mathrm{m}$ thick cryosections were prepared using SHANDON Cryotome E (Thermo Electron Corporation), placed on poly-L-lysine-coated slides, and air dried overnight. Slides were incubated with primary antibodies against the cleaved caspase-9/3 and PARP at $4{ }^{\circ} \mathrm{C}$ overnight and then with Alexa Fluor-555-conjugated secondary antibody in $1 \%$ BSA at room temperature for $2 \mathrm{~h}$, followed by counterstaining with DAPI. Slides were washed with PBS, and images were obtained at $40 \times$ under CLSM, processed using the Andor iQ2 software, and analyzed using the Image J software.

\section{Wound healing assay}

The near confluent B16-F10 monolayer was scratched using a sterile $200 \mu \mathrm{l}$ pipette tip. The wounds were subjected to media containing $500 \mathrm{nM}$ doxorubicin and $20 \mu \mathrm{M}$ LDCA alone or in combination and incubated at $37{ }^{\circ} \mathrm{C}$ with $5 \% \mathrm{CO}_{2}$ for $16 \mathrm{~h}$. Cells were stained with Giemsa, and the distance traversed was examined using a Nikon T $i$ phase contrast microscope at $20 \times$ and analyzed using the ImageJ software.

\section{Animal maintenance and melanoma tumor model}

All experiments were performed in accordance with the guidelines of the Committee for the Purpose of Control and Supervision of Experiments on Animals (CPCSEA), India, and as recommended by the animal ethics committee of CSIR-Indian Institute of Chemical Biology (Registration no. \# 147/1999/
CPCSEA). All possible efforts were made to minimize the suffering of animals and reduce the number of animals used. The animal care committee of CSIR-Indian Institute of Chemical Biology reviewed and approved the animal experiments. Adult female virgin BALB/c mice were issued from the institute and maintained under laboratory conditions (12:12, dark: light cycle); they were fed with a standard pellet diet and water ad libitum.

Then, $10^{6}$ melanoma cells were subcutaneously injected into the right flank to form a primary tumor. Mice were treated intravenously (IV) with $1 \mathrm{mg} \mathrm{kg}{ }^{-1}$ of doxorubicin, $2 \mathrm{mg} \mathrm{kg} \mathrm{kg}^{-1}$ LDCA, and their combination according to the dosing schedule after day 6 of the tumor challenge. Animals were sacrificed on the respective day, and tumors were excised for further studies.

\section{Statistical analysis}

All experiments were performed in triplicate, and data were representative of three independent experiments. For significance testing, unpaired two sample $t$-tests were used for comparisons between individual treatments and ANOVA with post hoc analysis for comparison among three or more groups, and Kaplan Meier was used for survival studies. ${ }^{*} p<0.05$ and $* * p<0.01$ were considered statistically significant. All statistical analyses were performed using the OriginPro 8 SR0 software.

\section{Results}

\section{Doxorubicin and LDCA combination synergistically perturbs melanoma cell viability in vitro}

Doxorubicin is extensively used in chemotherapeutic regimens; moreover, although its efficacy is poor in the treatment of human melanoma, the combination of doxorubicin with other chemotherapeutic agents has been significantly effective. ${ }^{36}$ LDCA is a dual-hit metabolic modulator that inhibits LDH-A enzyme activity to stimulate apoptosis in the malignant population; thus, to exploit the mechanism of relieving toxicity, we assessed the effect of LDCA in combination with doxorubicin in mitigating cancer. To assess the effect of LDCA and doxorubicin and the influence of their combination on the viability of melanoma cells, B16-F10 cells were incubated with LDCA (2$100 \mu \mathrm{M})$ and doxorubicin (10-500 $\mathrm{nM}$ ) for $72 \mathrm{~h}$, and the effects were studied using the MTT assay. The results show that $500 \mathrm{nM}$ of doxorubicin treatment evidently affected $\sim 40 \%$ melanoma viability, whereas treatment with $25 \mu \mathrm{M}$ of LDCA alone arrested $\sim 50 \%$ cell growth after $72 \mathrm{~h}$, demonstrating a dose-dependent cytotoxic effect against the cancerous population (Fig. 1A-C). Since both molecules demonstrated a significant cytotoxic effect, we extended our studies to decipher the doses at which both molecules would present reasonable additive or synergistic effect. Appreciably, the combination of $100 \mathrm{nM}$ of doxorubicin with $10 \mu \mathrm{M}$ of LDCA sufficiently inhibited $\sim 50 \%$ of melanoma viability; this comprehensively demonstrated the synergism of doxorubicin and LDCA (Fig. 1D). To precisely determine the nature of this combination, the median effect analysis of the Chou \& Talalay method was used. ${ }^{37}$ The CI values obtained from the dose range experiments data consequentially 
A
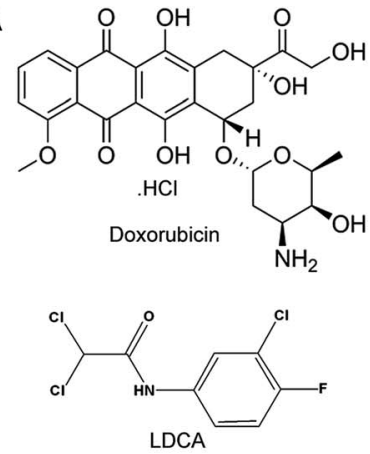

LDCA

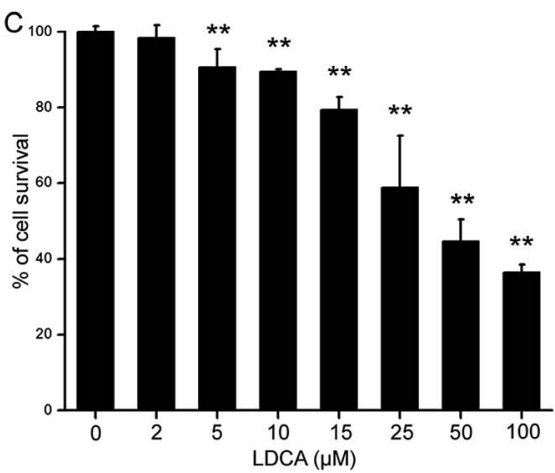

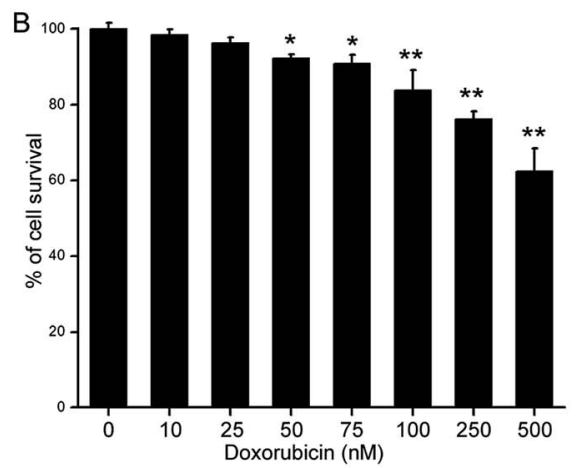

D

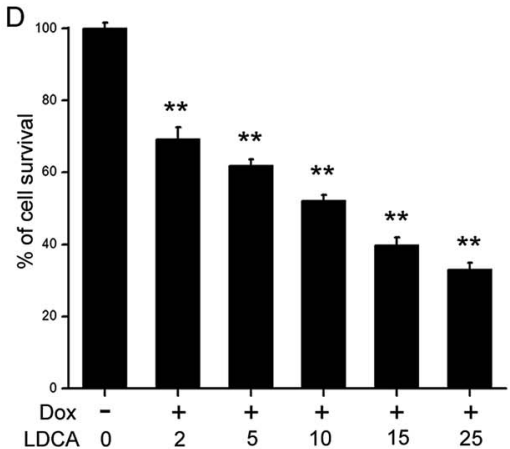

Fig. 1 Combinatorial synergism of doxorubicin and LDCA. (A) Chemical structure of doxorubicin and LDCA. Schematic representing \% of B16F10 cell viability upon treatment with (B) doxorubicin and (C) LDCA for $72 \mathrm{~h}$ using the MTT assay. $* *<0.01, * p<0.05$. (D) Melanoma cells were exposed to the combinations of $100 \mathrm{nM}$ of doxorubicin and varying doses of LDCA for $72 \mathrm{~h}$ and viability was assessed using the MTT assay. ${ }^{* *} p<$ 0.01. Error bars in all panels represent mean $\pm S D$.

ranged between 0.7 and 0.3 , signifying a strong synergism of doxorubicin and LDCA (Table 1 and ESI Fig. S1†). Importantly, with respect to the dose reduction index (DRI) values presented in Table 2 , for $50 \%$ melanoma growth inhibition, the doxorubicin dosage was significantly reduced by 5 -fold, and a 4 -fold decrease in the LDCA dose was documented.

\section{Combination of doxorubicin and LDCA realizes apoptosis and effectively suppresses clonogenic outgrowth}

Since the combination of doxorubicin and LDCA significantly generated a synergistic effect on cancer cells, we next determined whether combination treatment synergistically perturbs DNA to rationally subsidize apoptosis in the neoplastic population. The B16-F10 cells were exposed to $500 \mathrm{nM}$ of doxorubicin and $20 \mu \mathrm{M}$ of LDCA individually or in combination for $24 \mathrm{~h}$, and DNA strand breakage was assessed using the terminal deoxynucleotidyl transferase dUTP nick end labelling (TUNEL) assay technique. As depicted in Fig. 2A, compared to the vehicle control, $25 \%$ and $15 \%$ of TUNEL positive cells were noted in doxorubicin and LDCA treated sets, respectively; however, their combination significantly accounted for 55\% cells exhibiting DNA damage. To verify the postulation further, B16-F10 cells were treated with $500 \mathrm{nM}$ of doxorubicin and $20 \mu \mathrm{M}$ of LDCA either alone or in combination for $24 \mathrm{~h}$ and screened via flow cytometry using Annexin V-FITC to monitor cell membrane integrity. Cells exposed to doxorubicin and LDCA individually resulted in $18 \%$ and $15 \%$ death, respectively, whereas their combination synergistically caused $40 \%$ melanoma cell death
(Fig. 2B). Moreover, to visualize the apoptotic mode of cellular death, cells were stained with Hoechst and examined using a fluorescence microscope. The results demonstrated that combination treatment resultantly affected cellular morphology with condensed and fragmented nuclei (Fig. 2C). These data exemplify the role of doxorubicin and LDCA combination in inducing apoptotic death in malignant cells and stipulate the use of LDCA as a potential candidate in the therapeutic regimen.

Since the combinatorial regimen of doxorubicin and LDCA robustly induced apoptosis and resulted in significant inhibition of cell proliferation in the malignant cells, it was envisaged that the impact of combination treatment would adversely affect the clonogenic capability of the melanoma cells. ${ }^{38}$ Accordingly, treatment was initiated 3 days post seeding and continued every alternate day for 2 weeks. The colonies were examined using a phase contrast microscope, and sizes were

Table 1 Combination index $(\mathrm{Cl})$ values for doxorubicin and LDCA combination

\begin{tabular}{lcll}
$\begin{array}{l}\text { Doxorubicin } \\
(\mathrm{nM})\end{array}$ & LDCA $(\mu \mathrm{M})$ & Effect & CI \\
\hline 100.0 & 2.0 & 0.3 & 0.65173 \\
100.0 & 5.0 & 0.38 & 0.53628 \\
100.0 & 10.0 & 0.5 & 0.43034 \\
100.0 & 15.0 & 0.61 & 0.35256 \\
100.0 & 25.0 & 0.69 & 0.36702
\end{tabular}


Table 2 Dose reduction index (DRI) parameters for doxorubicin and LDCA

Fraction affected

(Fa) DRI LDCA DRI doxorubicin

\begin{tabular}{lcc}
\hline 0.3 & 10.7062 & 1.79107 \\
0.38 & 5.79772 & 2.74878 \\
0.5 & 4.38794 & 4.93972 \\
0.61 & 4.27237 & 8.43921 \\
0.69 & 3.45594 & 12.8753
\end{tabular}

analyzed to evaluate the tonic inhibitory responses of the combination treatment. Vehicle control B16-F10 cells formed large tumor spheres of about $275 \mu \mathrm{m}$ in diameter after 14 days; on the other hand, treatment with the combination of $500 \mathrm{nM}$ doxorubicin and $20 \mu \mathrm{M}$ of LDCA resulted in a significant regression of melanoma colony size to $52 \mu \mathrm{m}$ (Fig. 2D and E).
Conclusively, results suggest that the combination treatment initiates apoptosis and adversely prevents the tumor initiating ability of the melanoma cells.

\section{LDCA relieves the indiscriminate cytotoxicity of doxorubicin when used in combination}

Moreover, one of the major drawbacks of current chemotherapeutic regimens is the inability to precisely target cancer cells without side-effects. The combination of LDCA with doxorubicin leads to a significant 5-fold dose reduction of doxorubicin; moreover, since LDCA has a low toxicity profile, we decipher whether this combination would have any potential toxic manifestations on normal cells. Doxorubicin treatment usually leads to indiscriminate toxicity; however, the reason for this reduction in toxicity may be usage of the very low dose of doxorubicin that ultimately spares the cells from any undue off-
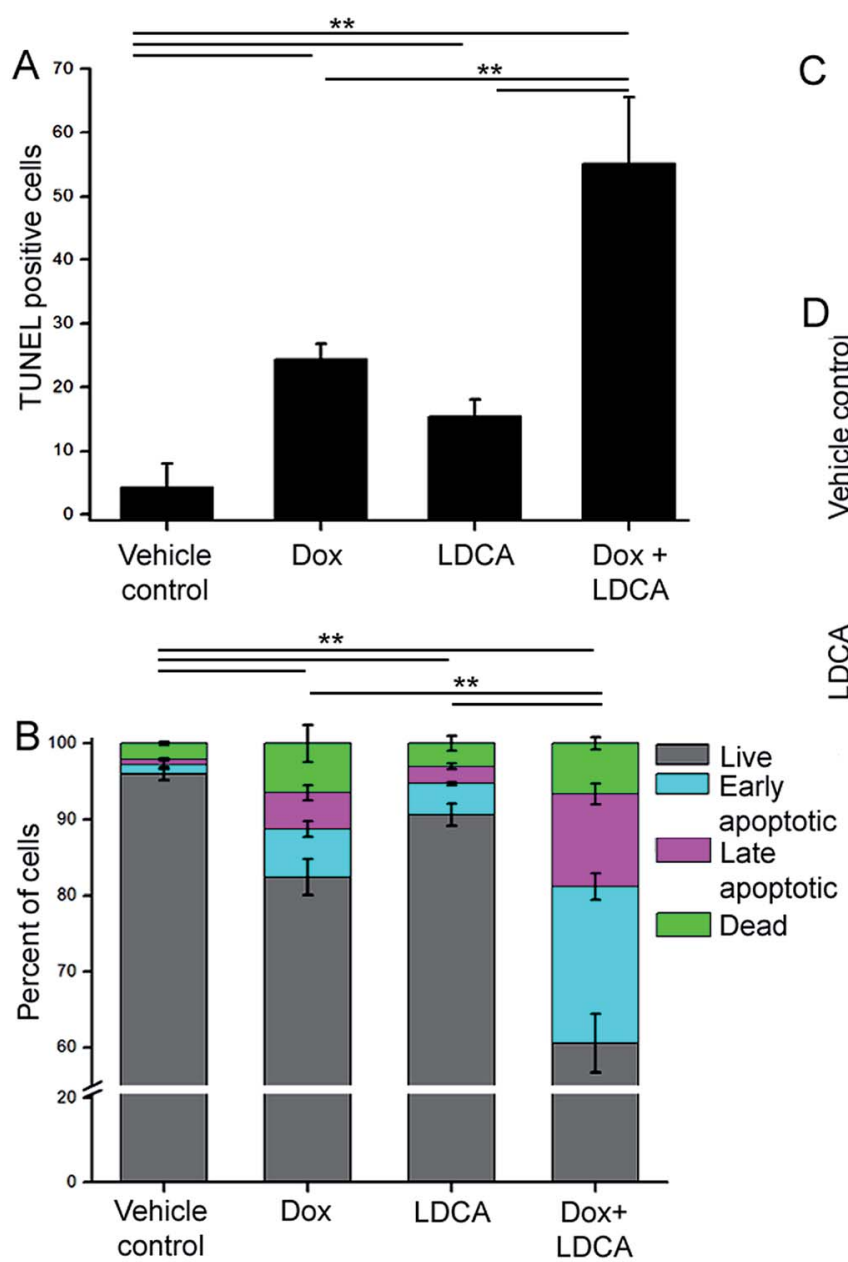

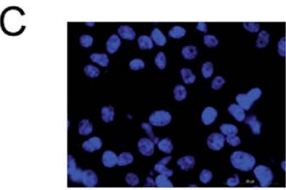

Vehicle control
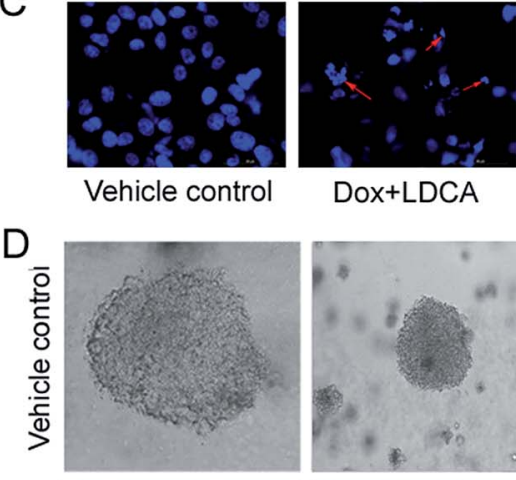

Dox+LDCA
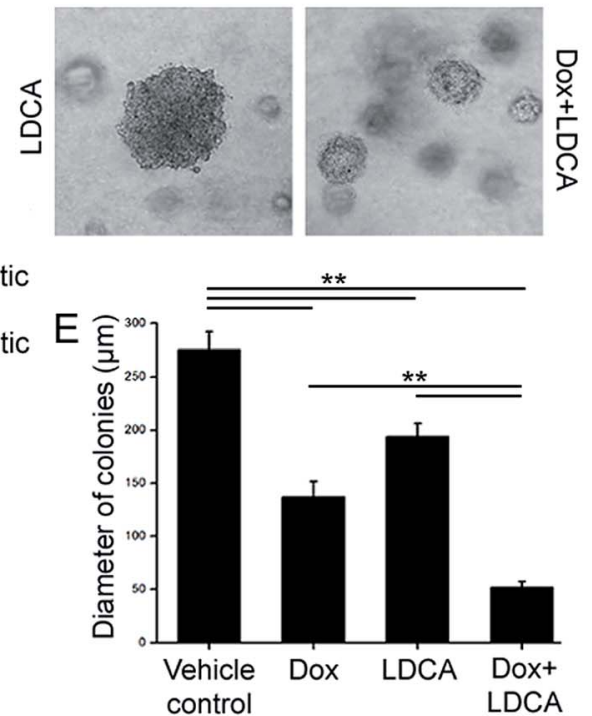

Fig. 2 Combination of doxorubicin and LDCA elicits apoptosis in the cancerous population. (A) Bars represent \% of TUNEL positive B16-F10 cells after $24 \mathrm{~h}$ in vehicle control, $500 \mathrm{nM}$ doxorubicin, $20 \mu \mathrm{M} \mathrm{LDCA}$, and their combination treated sets. **p< 0.01 . (B) Flow cytometric analysis of B16-F10 viability after $24 \mathrm{~h}$ of $500 \mathrm{nM}$ doxorubicin, $20 \mu \mathrm{M}$ LDCA treatment either alone or in combination using the Annexin V FITC/PI dual staining protocol. Stacked bars exhibit \% of cells that are viable, in early apoptotic, in late apoptotic phase or that are dead. **p< 0.01 . (C) Representative fluorescence images demonstrating the effect of $500 \mathrm{nM}$ doxorubicin and $20 \mu \mathrm{M}$ LDCA combination on B16-F10 cells. Arrows indicate condensed and fragmented nuclei. (D) Representative phase contrast images portraying the effect of treatment with $500 \mathrm{nM}$ doxorubicin, $20 \mu \mathrm{M}$ LDCA, alone or in combination on the sizes of colonies after 14 days of treatment. (E) Comparative quantitation shows the potential synergism of the combination in suppressing clonogenic outgrowth. ${ }^{* *} p<0.01$. Error bars in all panels represent mean \pm SD. 
target and side effects; furthermore, LDCA is selectively cytotoxic towards cancer cells. ${ }^{28}$ Stemming from the observation that combination treatment stringently disrupts the viability of B16-F10 cells, we similarly scrutinized the effect of treatment on the normal cells. WI-38 cells (human lung normal fibroblast) were treated with the selected combination of doxorubicin and LDCA, and their viability was analyzed using the MTT assay. Fig. 3A indicates that although the combination modulates a cytotoxic response towards the melanoma cells, this combination practically has no effect on the normal cell line. To confirm this further, flow cytometric analysis was performed using Annexin-V-FITC and propidium iodide (PI) after treating WI-38 cells with $500 \mathrm{nM}$ doxorubicin and $20 \mu \mathrm{M}$ LDCA combination for $24 \mathrm{~h}$; as expected, about $90 \%$ of fibroblasts were alive after treatment (Fig. 3B).

To plausibly understand the preferential mode of action of this combination, a straightforward method of study was employed where a co-culture of B16-F10 and WI-38 cells was treated with the combination of $500 \mathrm{nM}$ doxorubicin and $20 \mu \mathrm{M}$ LDCA. The effect of treatment has been visualized by employing confocal microscopy as B16-F10 cells produce melanin and exhibit contrasting morphology from that of WI-38 cells. Supposedly, the results depict that combination treatment specifically affects malignant cell viability, and normal cells are spared (Fig. 3C).

We next studied the effect of combination in expanding any potential cytotoxic manifestations on the peripheral blood lymphocytes. In this regard, human lymphocytes were treated with the combination of $100 \mathrm{nM}$ doxorubicin and $10 \mu \mathrm{M}$ LDCA for $72 \mathrm{~h}$, and the induction of chromosomal aberration and micronuclei formation, if any, was observed. The results reveal a low percentage of chromosomal aberration and micronuclei formation as compared to that of the positive control (Fig. 3D), signifying the potential of the combinatorial dosage in
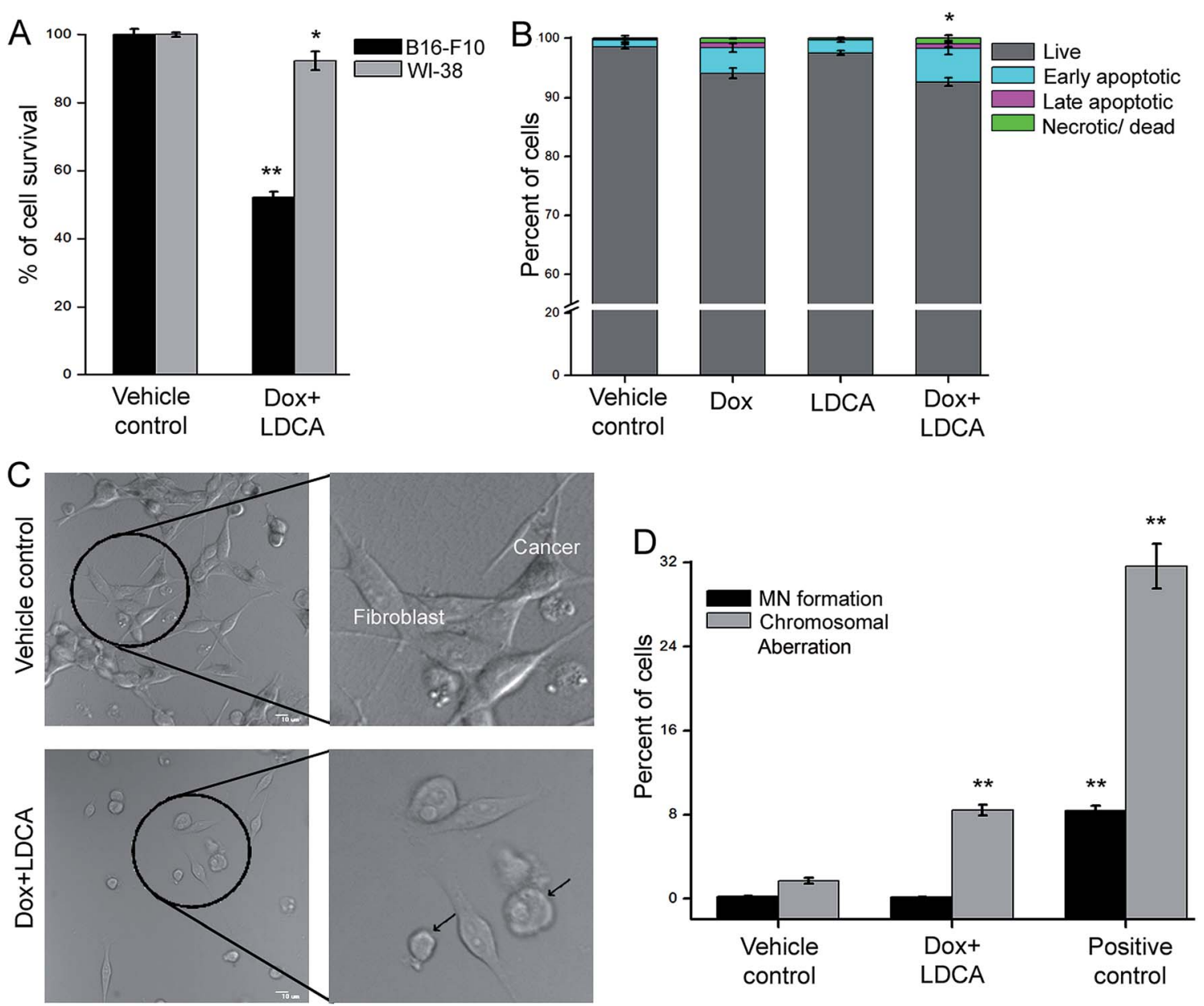

Fig. 3 LDCA combination sensitizes melanoma cells to doxorubicin treatment, obviating toxic manifestations. (A) Graph represents the effect of $100 \mathrm{nM}$ doxorubicin and $10 \mu \mathrm{M}$ LDCA combination treatment for $72 \mathrm{~h}$ on WI-38 in contrast to that on B16-F10 cells. Cell viability was estimated by employing the MTT assay. ${ }^{* *} p<0.01, * p<0.05$. (B) Graph represents flow cytometric analysis of WI-38 cells subjected to $500 \mathrm{nM}$ doxorubicin, $20 \mu \mathrm{M}$ LDCA either alone or in combination for $24 \mathrm{~h}$. Stacked bar graph represents the effect of treatment on the fibroblastic cells. * $p<0.05$. (C) The co-culture of B16-F10 and WI-38 cells was treated with the combination of $500 \mathrm{nM}$ doxorubicin and $20 \mu \mathrm{M}$ LDCA for $24 \mathrm{~h}$ and visualized using CLSM. Arrows indicate apoptotic cancerous cells. Right panel: enlarged view. (D) Graph representing the \% of micronuclei formation and chromosomal aberration induction in human peripheral lymphocytes post $72 \mathrm{~h}$ of $100 \mathrm{nM}$ doxorubicin and $10 \mu \mathrm{M}$ LDCA combination treatment.** $p<0.01$. Error bars in all panels represent mean \pm SD. 
mediating the selective killing of cancer, thus broadening the therapeutic window.

\section{The doxorubicin and LDCA combination affects mitochondrial hyperpolarization to augment caspase- mediated cell death}

Since doxorubicin and LDCA combination brought about a potentiated cytotoxic effect and equally compensated the toxic responses, we further elucidated its mechanistic pathway in mediating death of the malignant population. One of the hallmark characteristics of cancer cells is their resilient mitochondria. ${ }^{13,39}$ Offending mitochondrial hyperpolarization can be an effective strategy in curbing cancer-associated hallmark features; ${ }^{19}$ moreover, since LDCA essentially alters mitochondrial dynamics, we delineate whether this combination would have a factual impact and would be sufficiently effectual in degrading mitochondrial potential. ${ }^{40-42}$ B16-F10 cells were treated with the combination of $500 \mathrm{nM}$ doxorubicin and $20 \mu \mathrm{M}$ LDCA for $16 \mathrm{~h}$ and stained with JC1 dye; then, subsequent gradient shifts in membrane potential were observed using CLSM. Images revealed a significant mitochondrial depolarization in the treated sets as a high level of green and a low level of red fluorescence intensity was documented in contrast to that of the vehicle control (Fig. 4A). To substantiate the phenomenon of combinatorial synergism, flow cytometry was used, and the results indicated that individual treatment led to a decrease in the red:green fluorescence intensity ratio that further became significantly lowered following combination treatment; this confirmed the mitochondrial hyperpolarization alteration potential of the treatment (Fig. 4B). Additionally and importantly, to reasonably decipher whether the treatment affected the healthy population, WI-38 cells were subsequently treated with doxorubicin and LDCA for $16 \mathrm{~h}$ and subjected to flow cytometric analysis. ${ }^{29,43}$ As anticipated, combination treatment had minimal effect on the mitochondrial membrane potential of the fibroblastic population as the red : green fluorescence intensity ratio between the vehicle control and treated sets remained relatively unaltered; this comprehensively underlined the broad therapeutic window of the combinatorial regimen (Fig. 4C). After this, we studied whether degradation of mitochondrial polarization accounted for Cyt $\mathrm{c}$ release from the mitochondria as this event was documented to be intricately associated with mitochondrial damage. Immunofluorescence staining portrayed a distinct punctuate green staining pattern in the vehicle control, whereas combination treatment depicted a diffused staining pattern, referring to Cyt $\mathrm{c}$ dumping into the cytosol with relatively damaged mitochondria; this suggested that treatment with the combination of doxorubicin and LDCA ensured that cells reached the point of no return (Fig. 4D). Cyt c release triggers caspase activation; ${ }^{44}$ therefore, we studied the expression levels of the cleaved caspase $9 / 3$ proteins following combination treatment for $16 \mathrm{~h}$ in B16-F10 cells. The results strongly suggest that the treatment leads to heightened expression levels of the cleaved caspase 9/3, confirming the activation of the caspase cascade to ultimate apoptosis in the neoplastic population (Fig. 4E and F). Furthermore, consistent with the caspase $9 / 3$ results, the immunofluorescence study depicted a marked and significant expression of the cleaved poly (ADP-ribose) polymerase (PARP) in melanoma cells; this confirmed that combination treatment conferred mitochondrial apoptosis in the malignant population (Fig. 4G and ESI Fig. S2†).

\section{Doxorubicin and LDCA combination synergistically inhibits tumor progression in vivo}

The combination of doxorubicin and LDCA selectively and synergistically affects cancer cell viability as well as dampens the clonogenic outgrowing potential in soft agar; therefore, we have investigated the potential of this combination in vivo to comprehensively explicate the synergistic role of this combination in limiting the oncogenic progression. Syngeneic $10^{6}$ B16-F10 cells were injected subcutaneously into the right flank of immuno-competent female BALB/c mice, and after three days, intravenous (IV) injections of $1 \mathrm{mg} \mathrm{kg}^{-1}$ doxorubicin and $2 \mathrm{mg} \mathrm{kg}^{-1}$ LDCA, either alone or in combination, were administered according to the schedule via the lateral tail vein (ESI Fig. S3†). Comparative quantification showed that the combination treatment significantly accentuated survivability, causing a sustained limitation of tumor growth kinetics (Fig. 5A and B). Arguably, the fundamental trait for any therapeutic agent is to lend years to patient survival, and the results suggestively depict that tumor grows rapidly in the vehicle control cohort resulting in death of all the mice within 6 weeks; however, administration of doxorubicin and LDCA combination significantly increases mice survivability up to 8 weeks. Additionally, macroscopic examination of tumors shows widespread tumor necrosis and associated neovascularization in the vehicle control; on the other hand, the combination treatment effectively relieves mice from these tumor-associated phenomena, effectively establishing the role of combinatorial dose in restricting tumor progression (Fig. 5C).

Another phenotypic trait of the B16-F10 tumor is its dense vascularisation that helps it to accomplish the process of growth and consequently metastasis. An endothelium specific lectin (Ricinus communis agglutinin) was used to quantify tumor-associated neovasculature, and the results provided us a clear picture about the extent of vascularisation. Comparative quantification of lectin perfusion depicts that tumor vessel density is decreased post combination treatment as compared to that of the vehicle control sets (Fig. 5D: upper panel and ESI Fig. S4 $\uparrow$ ). Additionally, we evaluated the neovasculature density by staining the tissue sections with CD31, a marker extensively used to characterize endothelial cell specific traits. The results portray that microvessels have been scarcely detected in doxorubicin and LDCA-treated tumor sections as compared to the case of the vehicle control and suggestively state that treatment significantly reverses microvessel formation (Fig. 5D: lower panel and ESI Fig. S5†). Furthermore, the combinatorial regimen essentially modulates the phenomenon of angiogenesis to substantially limit oncogenic progression.

We further investigated whether the strategic and potential combination of doxorubicin and LDCA equally impacted the 


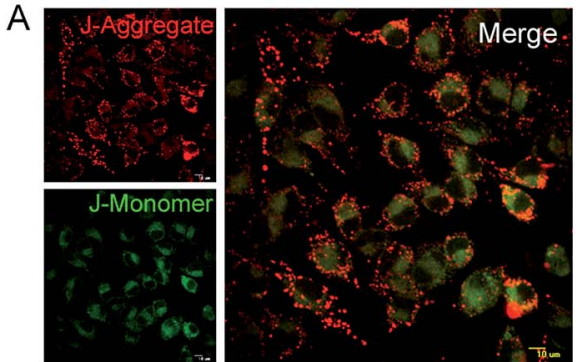

Vehicle control

C
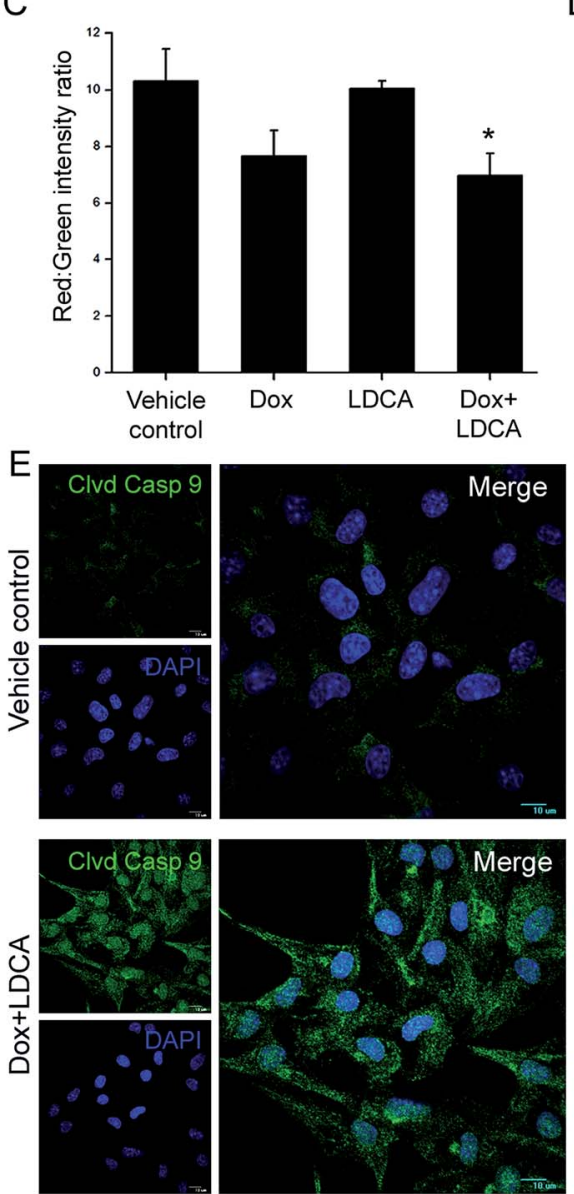

Cleaved Caspase 9

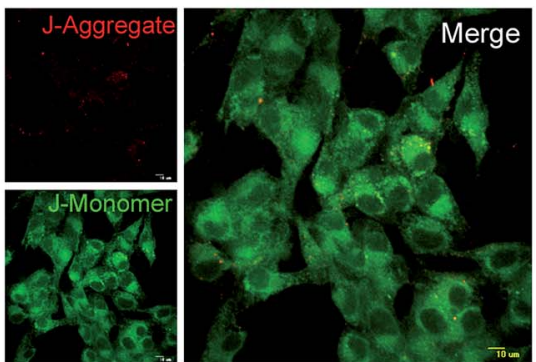

Dox+LDCA

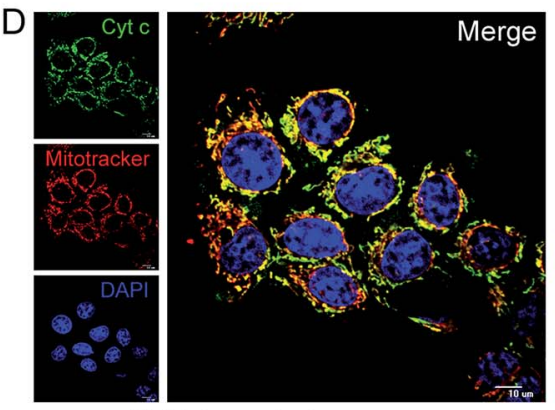

Vehicle control
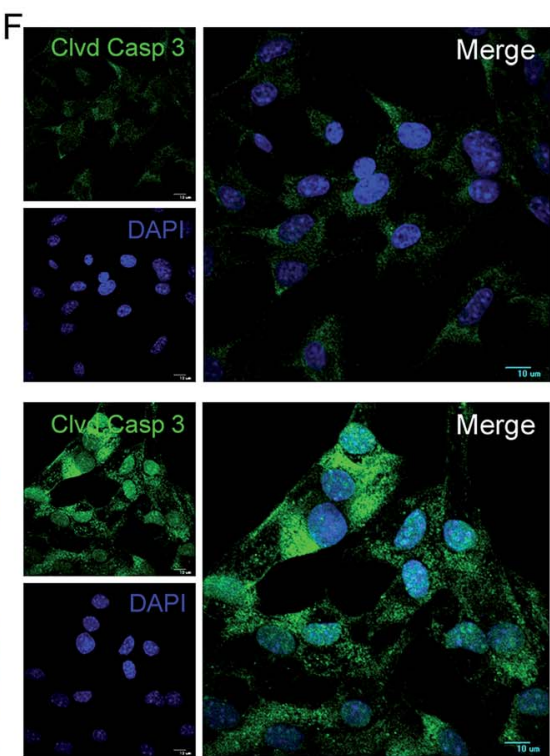

Cleaved Caspase 3
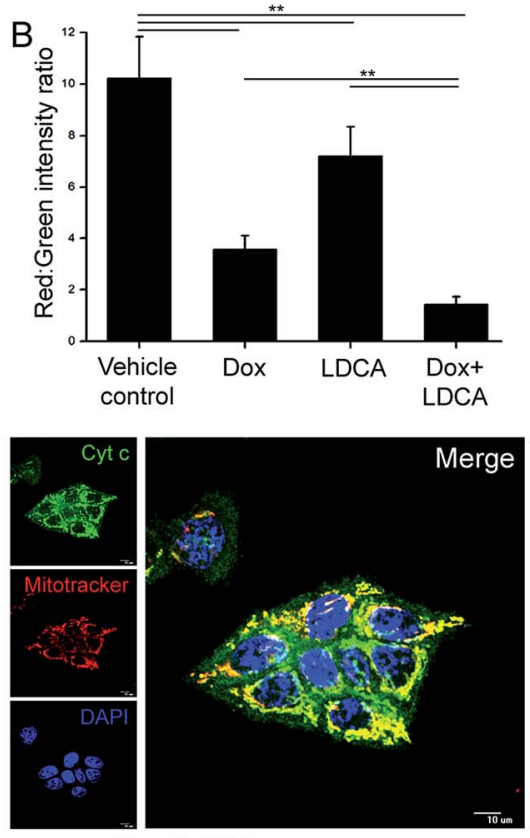

Dox+LDCA
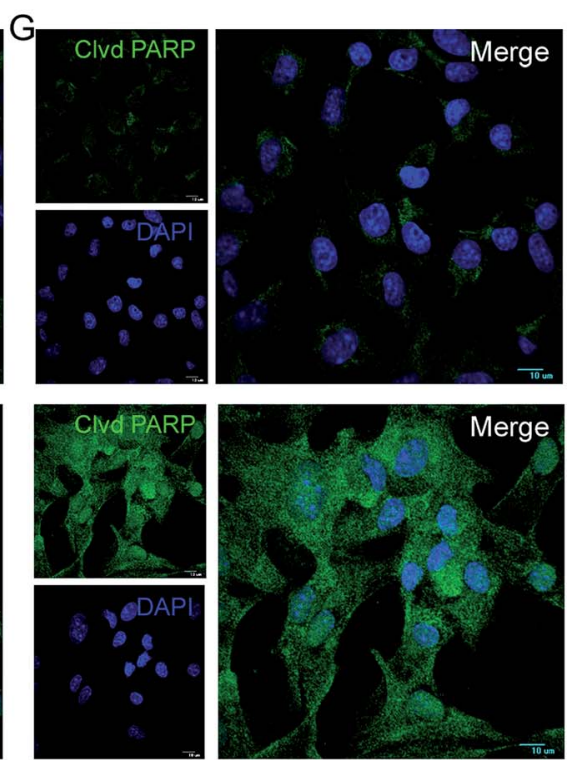

Cleaved PARP

Fig. 4 Doxorubicin and LDCA combination synergistically induces mitochondrial apoptosis selectively in the malignant cells. (A) Representative confocal microscopy images depicting degradation of mitochondrial hyperpolarization in B16-F10 cells post $16 \mathrm{~h}$ of $500 \mathrm{nM}$ doxorubicin and 20 $\mu M$ LDCA combination treatment. Heterogeneous red and green staining pattern was observed in vehicle control that was effectively reversed in the treated sets. Comparative quantification of the red:green fluorescence intensity ratio after treatment with the combination of $500 \mathrm{nM}$ doxorubicin and $20 \mu \mathrm{M}$ LDCA for $16 \mathrm{~h}$ in (B) B16-F10 and (C) WI-38 cells. ${ }^{*} p<0.01,{ }^{*} p<0.05$. (D) Representative confocal microscopy images depicting Cyt c release after $16 \mathrm{~h}$ in $500 \mathrm{nM}$ doxorubicin and $20 \mu \mathrm{M}$ LDCA combination-treated sets. Representative CLSM images portraying (E) cleaved casp- 9 (F) cleaved casp-3 and (G) cleaved PARP levels in vehicle control and $500 \mathrm{nM}$ doxorubicin and $20 \mu \mathrm{M}$ LDCA combination treated B16-F10 cells (right panel: enlarged view of merged images). Error bars in all panels represent mean \pm SD.

mitochondrial hyperpolarization of tumor cells in vivo. Freshly resected tumor tissues from experimental cohorts were stained with JC1 dye and analyzed employing CLSM. The results portrayed a significant dissipation of mitochondrial potential as the combination treatment resulted in a 3 -fold decrease in the red : green fluorescence intensity ratio as compared to that of the vehicle control (ESI Fig. S6†). Since the combination effectively abrogated mitochondrial membrane potential within the tumor, we posited that it would effectively meditate apoptosis in vivo. To comprehensively outline the phenomenon of apoptosis, vehicle control and combination-treated tumors were resected and sectioned, and fluorescence immunohistochemical analysis was performed. The results showcased that mice subjected to combinatorial treatment 

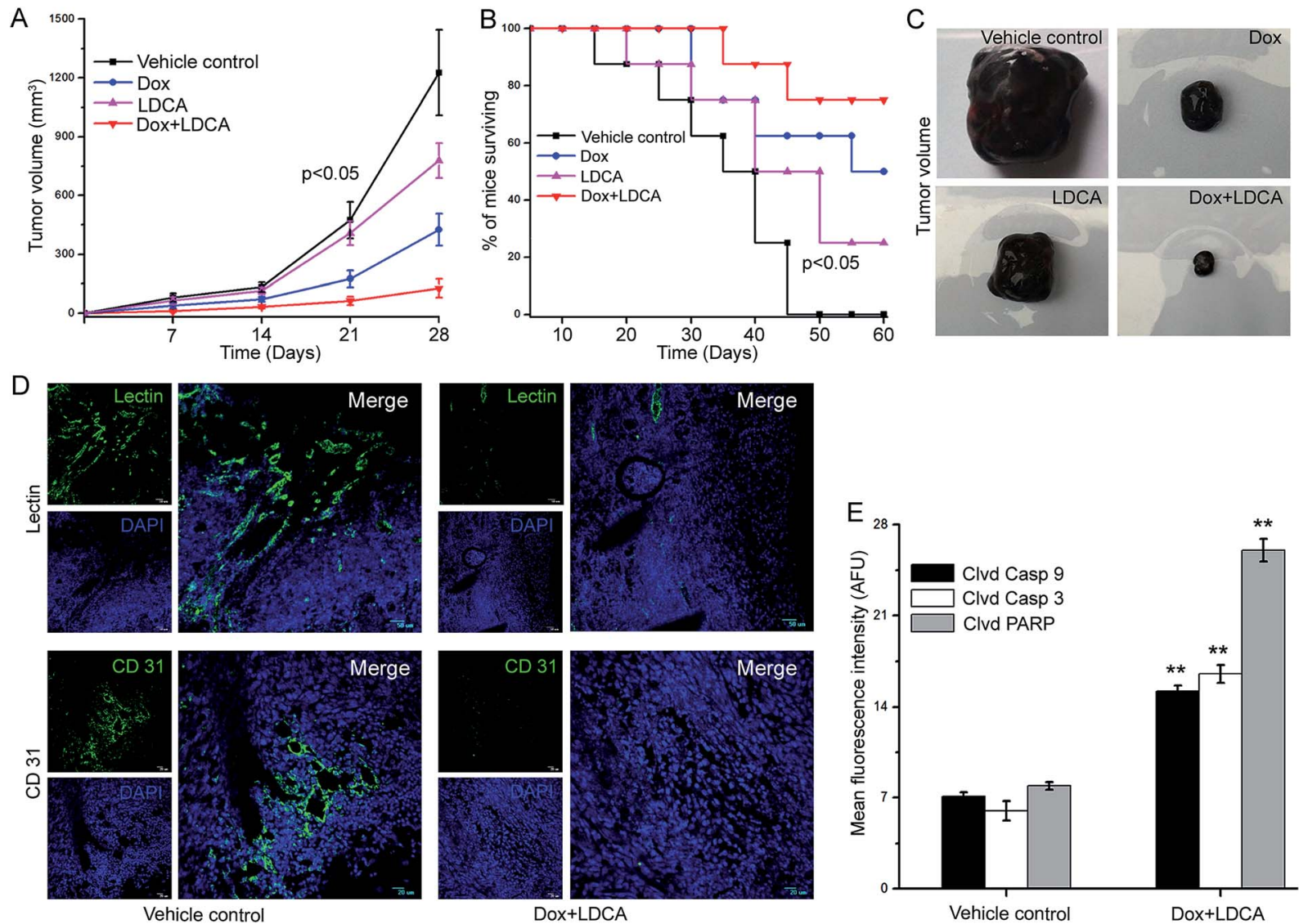

Fig. 5 Combination treatment synergistically attenuates tumor progression in vivo. Intravenous administration of $1 \mathrm{mg} \mathrm{kg}^{-1} \mathrm{doxorubicin}$ and $2 \mathrm{mg} \mathrm{kg}^{-1}$ LDCA combination decreases (A) tumor volume and (B) increases survivability in B16-F10 tumor burdened BALB/c mice as compared to that of vehicle control and individual components. Graph portrays the potential synergism in combination treated cohorts. * $p<0.05$ between vehicle control and treated sets. ${ }^{*} p<0.05$ between single treatment and combination treated sets. (C) Melanoma tumor bearing mice were sacrificed on day 28; tumors were excised, and imaged, and the representative images have been presented. (D: upper panel) 1 mg $\mathrm{kg}^{-1}$ doxorubicin and $2 \mathrm{mg} \mathrm{kg}^{-1}$ LDCA combination treatment affects neovasculature formation as immunohistochemical analysis indicates decreased lectin signal intensity. (Right panel: enlarged view of merged images). (D: lower panel) representative CLSM images depicting CD31 staining in vehicle control and combination treated sets. (Right panel: enlarged view of merged images). (E) Graphical analysis demonstrating quantitative levels of cleaved caspase $9 / 3$ and PARP levels in vehicle control and $1 \mathrm{mg} \mathrm{kg}^{-1}$ doxorubicin and $2 \mathrm{mg} \mathrm{kg}^{-1} \mathrm{LDCA}_{\mathrm{c}} \mathrm{combination}$ treated sets. ${ }^{* *} p<0.01$. Error bars in all panels represent mean $\pm \mathrm{SD}$

had a 2.1-, 2.7- and a 3.2-fold increase in the cleaved caspase 9/ 3 and PARP expression levels, respectively, as compared to the vehicle control (Fig. 5E and ESI Fig. S7†). Convincingly, the combination of doxorubicin and LDCA combats tumor growth by triggering the caspase cascade.

\section{Combination of doxorubicin and LDCA alters cytoskeletal dynamics to arrest the migratory potential of melanoma cells}

The process of tumor progression involves the phenotypical characterizations of migration and invasion. Directional migration is a featured trait essentially related to metastasis; to investigate the impact of doxorubicin and LDCA combination treatment on this phenotypic aspect, a wound healing assay was performed. The treatment significantly limited the migratory potential of the B16-F10 cells, and comparative quantification suggestively depicted the synergistic inhibitory potential of doxorubicin and LDCA combination in restraining the migratory ability of cancer cells (Fig. 6A and B). The phenomenon of migration continually demands actin reorganization and microtubule remodeling; moreover, since treatment levied a limitation on the migratory ability of the cancer cells, we next assessed whether treatment potentially affected cytoskeletal dynamics. Treatment with a combination of $500 \mathrm{nM}$ doxorubicin and $20 \mu \mathrm{M}$ LDCA substantially compromises F-actin assembly and acetylated tubulin recruitment on the migrating front to essentially slow down the microtubule turnover (Fig. 6C). Results suggest the synergistic potential of LDCA and doxorubicin combination to limit the metastatic potential of the tumor cells and factually comprehend the significance of this combination in chronically inhibiting the crucial stages of oncogenic progression.

\section{Discussion}

Despite the availability of several approved therapeutics, metastatic melanoma remains a challenge. ${ }^{1-3}$ The increase in 

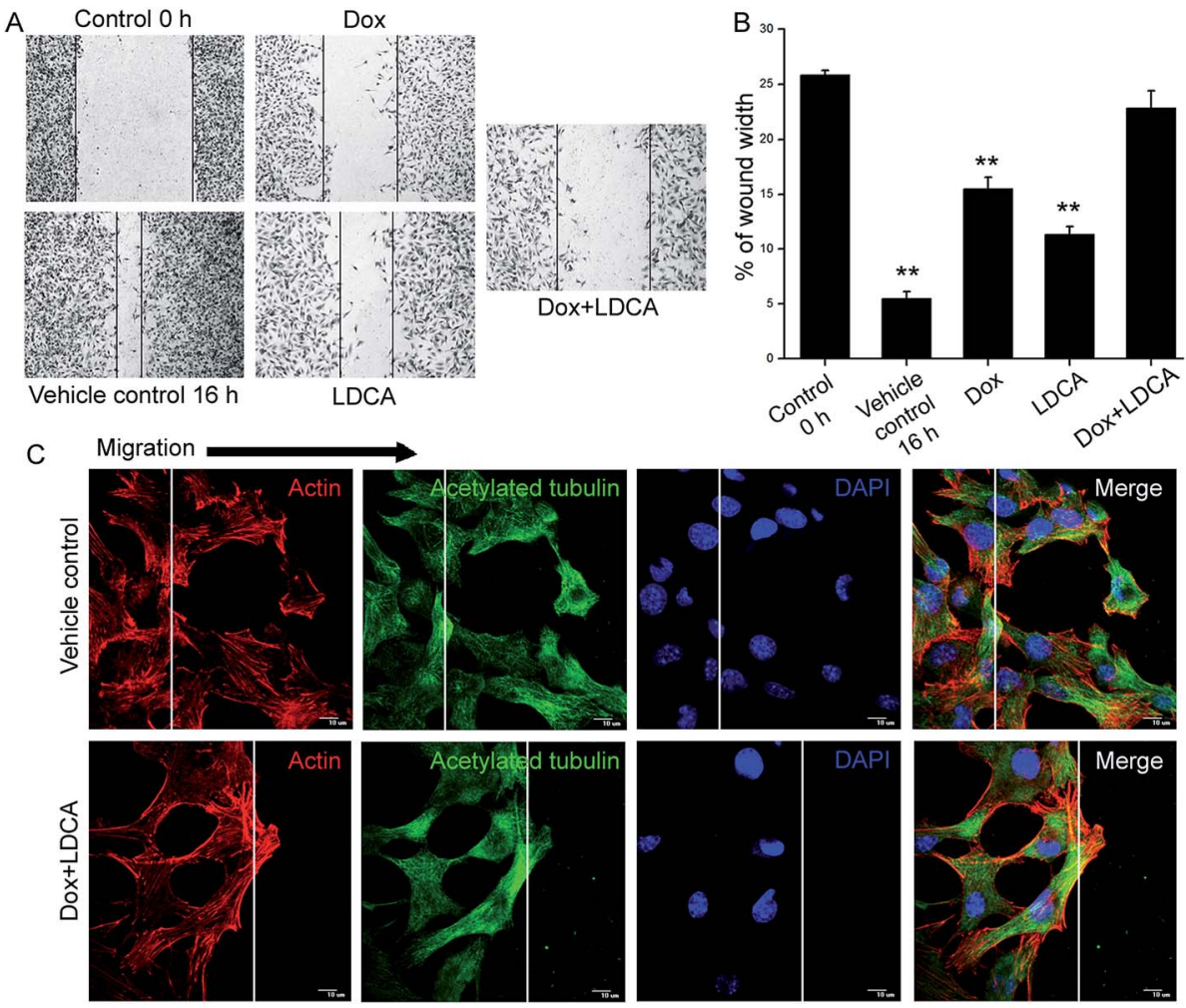

Fig. 6 Combination treatment modulates cytoskeletal dynamics to inhibit migratory behavior of cancer cells (A) representative wound healing images depicting the ability of $500 \mathrm{nM}$ doxorubicin, $20 \mu \mathrm{M} \mathrm{LDCA}$, either alone or in combination treatment in obviating the migratory ability of melanoma cells after $16 \mathrm{~h}$. (B) Comparative quantification exemplifying the wound healing rates with respect to $0 \mathrm{~h}$ vehicle control. **p< 0.01 . (C) Representative images demonstrating the effect of $500 \mathrm{nM}$ doxorubicin and $20 \mu \mathrm{M}$ LDCA combination in restricting lamellipodia formation and directional cell polarization. White lines represent wound edges; arrows indicate actin ruffles and microtubule organization center (MTOC). Cells were stained for f-actin (red), acetylated tubulin (green), and nucleus (blue). Error bars in all panels represent mean \pm SD.

systemic toxicity and drug resistance is the major impediment in the field of cancer therapeutics; thus, to overcome this obstacle, extensive research is being directed towards the design of rational combinatorial therapy that would not only elevate the therapeutic index but also broaden the therapeutic window with reduced toxicity. Doxorubicin as well as other established anthracycline chemotherapeutic agents potentially display high toxicity levels that cause adverse health effects such as immunosuppression and cardiomyopathy, leading to dose restrictions that concomitantly may lead to the development of primary or secondary drug resistance in tumor cells; this limits their therapeutic success. ${ }^{\mathbf{8}, 9,45}$ Recently, there have been significant advances in the understanding of the molecular mechanisms underlying the metabolic transformations in cancer and the role of mitochondrial bioenergetics and subsequent alterations in neoplastic transformations. Since metabolic reprogramming is a characteristic of cancer cells, emerging evidence provide a strong rationale for the use of metabolic modulators in combination therapy. Our studies have shown that the dualhit metabolic modulator LDCA is one such agent that has a low toxicity profile and no potential off-target effects, but effectively inhibits oncogenic progression both in vitro and in vivo in aggressive breast cancer cells. In this study, we have focused on the development of combination-targeted therapies against skin cancer, and the central finding stemming from this study is that LDCA potentially improves the therapeutic efficacy of doxorubicin in advanced melanoma via metabolic alteration and caspase-mediated cell death. On the basis of our prior studies and the fact that doxorubicin-caused systemic toxicity poses a major challenge in maximizing the beneficial outcome of doxorubicin therapy in melanoma patients, we hypothesized that using a combination approach of LDH-A inhibitor, LDCA with a DNA synthesis inhibitor, doxorubicin would garner a strong synergistic efficacy against metastatic melanoma. In this regard, the results indicate that LDCA interacts synergistically with doxorubicin to induce apoptosis of the melanoma population in vivo and in vitro. We report that the combination of LDCA and doxorubicin with reduced dosage tightly controls cancer progression without affecting the normal population and thus broadens the therapeutic window. LDCA and doxorubicin treatment synergistically shifts the mitochondrial membrane potential towards degradation of its hyperpolarized state; this allows Cyt c dumping from the mitochondria. Further, the synergistic repercussion was validated as an increase in the levels of cleaved caspase $9 / 3$ and PARP was documented upon treatment, favoring apoptosis. Importantly, in contrast to regular chemotherapy, this alternative approach exerts its effects by targeting the metabolic pathway that is 
crucial for the sustenance of the cancerous population. Since it effectively inhibited cancer growth and proliferation in vitro, we next sought to understand whether this regimen would have an equally durable impact in vivo towards mitigating cancer. Intravenous administration of scalar doses of LDCA and doxorubicin in a syngeneic model of murine melanoma effectively relieved tumor burden and increased survivability in contrast to the cohort of vehicle control and individual counterparts. Furthermore, preclinical data obtained from these studies comprehensively suggest that the combination treatment sufficiently obviates neo-vasculature formation, degrades mitochondrial membrane potential, and critically capsizes the initiation of the caspase cascade to substantiate the apoptotic trigger in vivo.

\section{Conclusion}

In this study, we have combined two anti-cancer drugs with disparate mechanisms of action; this is a feasible strategy to increase the therapeutic efficacy while avoiding unacceptable side effects. Consistent with this postulation, the combination of doxorubicin with LDCA aggravates synergistic cytotoxicity specifically towards cancer cells while sparing the normal cells. This indicates the great application potential and relevance of the doxorubicin-LDCA combination in a clinical setting.

\section{Conflicts of interest}

The authors declare that there are no conflicts of interest.

\section{Acknowledgements}

The authors thank the Director (CSIR-IICB) for continuous support. S. S. and M. G. acknowledges CSIR and UGC (Govt. of India) for providing fellowships. This work was supported by the CSIR Network Project No. 31-2(112)/NWP09, CSIR-IICB Director's seed money and the contingency grant of S. S. and M. G.

\section{References}

1 H. I. Hall, D. R. Miller, J. D. Rogers and B. Bewerse, J. Am. Acad. Dermatol., 1999, 40, 35-42.

2 W. Weyers, M. Euler, C. Diaz-Cascajo, W. B. Schill and M. Bonczkowitz, Cancer, 1999, 86, 288-299.

3 H. R. Byers and J. Bhawan, Clin. Haematol., 1998, 12, 717735.

4 K. M. McMasters, V. K. Sondak, M. T. Lotze and M. I. Ross, Ann. Surg. Oncol., 1999, 6, 467-475.

5 O. Tacar, P. Sriamornsak and C. R. Dass, J. Pharm. Pharmacol., 2013, 65, 157-170.

6 G. N. Hortobagyi, Drugs, 1997, 54, 1-7.

7 R. H. Blum and S. K. Carter, Ann. Intern. Med., 1974, 80, 249259.

8 N. Y. Frank, A. Margaryan, Y. Huang, T. Schatton, A. M. Waaga-Gasser, M. Gasser, M. H. Sayegh, W. Sadee and M. H. Frank, Cancer Res., 2005, 65, 4320-4333.
9 A. M. Elliott and M. A. Al-Hajj, Mol. Cancer Res., 2009, 7, 7987.

10 E. Berman, E. S. Casper, J. Howard and R. E. Wittes, Cancer Treat. Rep., 1984, 68, 675-677.

11 D. A. Vorobiof, B. L. Rapoport, R. Mahomed and M. Karime, Melanoma Res., 2003, 13, 201-203.

12 O. Warburg, K. Posener and E. Negelein, Biochem. Z., 1924, 152, 319-344.

13 G. Kroemer and J. Pouyssegur, Cancer Cell, 2008, 13, 472482.

14 D. Hanahan and R. A. Weinberg, Cell, 2011, 144, 646-674.

15 C. V. Dang, Genes Dev., 2012, 26, 877-890.

16 P. P. Hsu and D. M. Sabatini, Cell, 2008, 134, 703-707.

17 M. G. Vander Heiden, L. C. Cantley and C. B. Thompson, Science, 2009, 324, 1029-1033.

18 Y. Zhao, E. B. Butler and M. Tan, Cell Death Dis. Nerv. Syst., 2013, 4, e532, DOI: 10.1038/cddis.2013.60.

19 S. E. Weinberg and N. S. Chandel, Nat. Chem. Biol., 2015, 11, 9-15.

20 J. G. Pan and T. W. Mak, Sci. Signaling, 2007, 381, pe14.

21 M. G. Vander Heiden, Nat. Rev. Drug Discovery, 2011, 10, 671684.

22 L. Galluzzi, O. Kepp, M. G. Vander Heiden and G. Kroemer, Nature Review Drug Discovery, 2013, 12, 829-846.

23 D. A. Tennant, R. V. Durãn and E. Gottelieb, Nat. Rev. Cancer, 2010, 10, 267-277.

24 H. Shim, C. Dolde, B. C. Lewis, C. S. Wu, G. Dang, R. A. Jungmann, R. Dalla-Favera and C. V. Dang, Proc. Natl. Acad. Sci. U. S. A., 1997, 94, 6658-6663.

25 V. R. Fantin, J. St-Pierre and P. Leder, Cancer Cell, 2006, 9, 425-434.

26 A. Le, C. R. Cooper, A. M. Gouw, R. Dinavahi, A. Maitra, L. M. Deck, R. E. Royer, D. L. Vander Jagt, G. L. Semenza and C. V. Dang, Proc. Natl. Acad. Sci. U. S. A., 2010, 107, 2037-2042.

27 H. Xie, V. A. Valera, M. J. Merino, A. M. Amato, S. Signoretti, W. M. Linehan, V. P. Sukhatme and P. Seth, Mol. Cancer Ther., 2009, 8, 626-635.

28 M. Ghosh, S. Saha and S. K. Dutta, Chem. Commun., 2016, 52, 2401-2404.

29 M. Ghosh, S. Saha and S. K. Dutta, RSC Adv., 2016, 6, 6645766467.

30 R. W. Brockman, Adv. Cancer Res., 1963, 7, 129-234.

31 R. T. Schimke, R. J. Kaufman, F. W. Alt and R. F. Kellems, Science, 1978, 202, 1051-1055.

32 M. M. Gottesman, Annu. Rev. Med., 2002, 53, 615-627.

33 V. T. DeVita Jr, R. C. Young and G. P. Canellos, Cancer, 1975, 35, 98-110.

34 B. A. Chabner and T. G. Roberts Jr, Nat. Rev. Cancer, 2005, 5, 65-72.

35 B. Al-Lazikani, U. Banerji and P. Workman, Nat. Biotechnol., 2012, 30, 679-692.

36 B. Jose, L. Norton, J. Albanell, Y. M. Kim and J. Mendelsohn, Cancer Res., 1998, 58, 2825-2831.

37 T. C. Chou, Cancer Res., 2010, 70, 440-446. 
38 S. Mori, J. T. Chang, E. R. Andrechek, N. Matsumura, T. Baba, G. Yao, J. W. Kim, M. Gatza, S. Murphy and J. R. Nevins, Oncogene, 2009, 28, 2796-2805.

39 J. Henry-Mowatt, C. Dive, J. Martinou and D. James, Oncogene, 2004, 23, 2850-2860.

40 M. Huigsloot, I. B. Tijdens, G. J. Mulder and B. van de Water, J. Biol. Chem., 2002, 277, 35869-35879.

41 A. Malugin, P. Kopeèková and J. Kopeèek, Mol. Pharm., 2006, 3, 351-361.
42 E. Synowiec, G. Hoser, J. Bialkowska-Warzecha, E. Pawlowska, T. Skorski and J. Blasiak, BioMed Res. Int., 2015, 2015, 673512.

43 M. E. Stearns, M. D. Amatangelo, D. Varma, C. Sell and S. M. Goodyear, Am. J. Pathol., 2010, 177, 3169-3179.

44 E. A. Slee, M. T. Harte, R. M. Kluck, B. B. Wolf, C. A. Casiano, D. D. Newmeyer, H. G. Wang, J. C. Reed, D. W. Nicholson, E. S. Alnemri and D. R. Green, J. Cell Biol., 1999, 144, 281292.

45 S. M. Wain, F. S. Whaley and M. S. Ewer, Cancer, 2003, 97, 2869-2879. 